\title{
Matrix Transformation into a New Sequence Space Related to Invariant Means
}

\author{
Adnan Alhomaidan \\ Department of Mathematics, Faculty of Science, \\ King Abdulaziz University \\ P.O. Box 80081, Jeddah 21589, Saudi Arabia \\ alhomaidana@yahoo.com
}

\begin{abstract}
In this paper we define a sequence space $V_{\infty}$ through the concept of invariant means and prove that this is a Banach space under certain norm. We further characterize the matrix classes $\left(l_{\infty}\right.$, $\left.V_{\infty}\right)$ and $\left(l_{1}, V_{\infty}\right)$.
\end{abstract}

AMS subject classification: 40H05, 46A45.

Keywords: Sequence spaces, invariant mean, matrix transformations.

\section{Introduction and Preliminaries}

Let $l_{\infty}$ and $c$ be the Banach spaces of bounded and convergent sequences $x=$ $\left(x_{k}\right)$ respectively with norm $\|x\|_{\infty}=\sup _{k \geq 0}\left|x_{k}\right|$, and $l_{1}$ be the space of absolutely convergent series with $\|x\|_{1}=\sum_{k}\left|x_{k}\right|$.

Let $\sigma$ be a mapping of the set of positive integers $\mathbb{N}$ into itself. A continuous linear functional $\phi$ on $l_{\infty}$ is said to be an invariant mean or a $\sigma$-mean if and only if, (i) $\phi(x) \geq 0$ when the sequence $x=\left(x_{k}\right)$ has $x_{k} \geq 0$ for all $k$, (ii) $\phi(e)=1$, where $e=(1,1,1, \cdots)$, and (iii) $\phi(T x)=\phi(x)$ for all $x \in l_{\infty}$, where $T x=\left(T x_{k}\right)=$ $\left(x_{\sigma(k)}\right)$. In case $\sigma$ is the translation mapping $k \rightarrow k+1$, a $\sigma$-mean is often called a Banach limit $\left.{ }^{1]}\right]$ and $V_{\sigma}$, the set of bounded sequences all of whose invariant means are equal, is the set $f$ of almost convergent sequences ${ }^{[2]}$.

Note that ${ }^{[3]}$,

$$
V_{\sigma}:=\left\{x \in l_{\infty}: \lim _{m} t_{m n}(x)=L \text { uniformly in } n, L=\sigma-\lim x\right\},
$$


where

$$
t_{m n}(x)=\left(x_{n}+T x_{n}+\cdots+T^{m} x_{n}\right) /(m+1),
$$

and

$$
t_{-1, n}=0 \text {. }
$$

A $\sigma$-mean extends the limit functional on $c$ in the sense that $\phi(x)=\lim x$ for all $x \in c$ if and only if $\sigma$ has no finite orbits, that is to say, if and only if, for all $n \geq 0, m \geq 1, \sigma^{m}(n) \neq n$ (see Ref. [4]).

We say that a bounded sequence $x$ is $\sigma$-convergent if and only if $x \in V_{\sigma}$ such that $\sigma^{m}(n) \neq n$ for all $n \geq 0, m \geq 1$ (see Ref. [5]).

Let $X$ and $Y$ be two sequence spaces and $A=\left(a_{n k}\right)_{n, k=1}^{\infty}$ be an infinite matrix of real complex numbers. We write $A x=\left(A_{n}(x)\right)$ where $A_{n}(x)=\sum_{k} a_{n k} x_{k}$ and the series converges for each $n$. If $x=\left(x_{k}\right) \in X$ implies that $A x \in Y$, then we say that $A$ defines a matrix transformation from $X$ into $Y$. By $(X, Y)$ we denote the class of matrices $A$ such that $A x \in Y$ for $x \in X$.

In this paper we define a new sequence space $V_{\infty}$ related to the concept of $\sigma$ mean and prove that $V_{\infty}$ in a Banach space under certain norm. We also characterize the matrices of the class $\left(l_{\infty}, V_{\infty}\right)$ and $\left(l_{1}, V_{\infty}\right)$.

We define the space $V_{\infty}$ as follows

$$
V_{\infty}:=\left\{x \in l_{\infty}: \sup _{m, n}\left|t_{m n}(x)\right|<\infty\right\} .
$$

Note that if $\sigma$ is a translation then $V_{\infty}$ is reduced to the space

$$
f_{\infty}:=\left\{x \in l_{\infty}: \sup _{m, n}\left|g_{m n}(x)\right|<\infty\right\} .
$$

where

$$
g_{m n}(x)=\frac{1}{m+1} \sum_{k=0}^{\infty} x_{k+n} .
$$

We call the space $V_{\infty}$ as the space of $\sigma$-bounded sequences. It is clear that $c \subset V_{\sigma} \subset V_{\infty} \subset l_{\infty}$.

\section{Results}

\section{Theorem 1}

$V_{\infty}$ is a Banach space normed by

$$
\|x\|=\sup _{m, n}\left|t_{m n}(x)\right|
$$




\section{Proof}

It is easy to see that $V_{\infty}$ is a normed linear space under the norm in (1).

Now we have to show the completeness of $V_{\infty}$. Let $\left(x^{(i)}\right) \underset{i=1}{\infty}$ be a Cauchy sequence in $V_{\infty}$. Then $\left(x_{k}^{(i)}\right) \underset{i=1}{\infty}$ is Cauchy sequence in $\mathbb{R}$ for each $k$ and hence convergent in $\mathbb{R}$ that is, $x_{x}^{(i)} \rightarrow x_{k}$, say, as $i \rightarrow \infty$. Let $x=\left(x_{k}\right)_{k=1}^{\infty}$. Then by the definition of norm on $V_{\infty}$, we can easily show that

$$
\left\|x^{(i)}-x\right\| \rightarrow 0 \text { as } i \rightarrow \infty \text {. }
$$

Now, we have to show that $x \in V_{\infty}$. Since $\left(x^{(i)}\right.$ is a Cauchy sequence, given $\varepsilon$ $>0$, there is a positive integer $N$ depending upon $\varepsilon$ such that, for each $i, r>N$,

$$
\left\|x^{(i)}-x^{(r)}\right\| \varepsilon
$$

Hence

$$
\sup _{m, n}\left|t_{m n}\left(x^{(i)}-x^{(r)}\right)\right|<\varepsilon .
$$

This implies that

$$
\mid t_{m n}\left(x^{(i)}-x^{(r)} \mid<\varepsilon,\right.
$$

for each $m, n$; or

$$
\left|L^{(i)}-L^{(r)}\right|<\varepsilon
$$

for each $i, r>N$; where $L^{(i)}=\sigma-\lim x^{(i)}$. Let $L=\lim _{r \rightarrow \infty} L^{(r)}$. Then the $\sigma$ mean of $x, \phi(x)=\lim _{i} \phi\left(x^{(i)}\right)=\lim _{i} L^{(i)}=L$. Letting $r \rightarrow \infty$ in (2) and (3), we get

$$
\left|t_{m n}\left(x^{(i)}-x\right)\right| \leq \varepsilon, \text { for each } m, n
$$

and

$$
\left|L^{(i)}-L\right| \leq \varepsilon
$$

for $i>N$. Now, fix $i$ in the above inequalities. Since $x^{(i)} \in V_{\infty}$ for fixed $i$, we obtain

$$
\lim _{m} t_{m n}\left(x^{(i)}\right)=L^{(i)} \text {, uniformly in } n .
$$

Hence, for a given $\varepsilon$, there exists a positive integer $m_{0}$ (depending upon $i$ and $\varepsilon$ but not on $n$ ) such that

$$
\mid t_{m n}\left(x^{(i)}-L^{(i)} \mid<\varepsilon,\right.
$$

for $m \geq m_{0}$ for all $n$. Now, by (4), (5) and (6), we get 


$$
\left|t_{m n}(x)-L\right| \leq\left|t_{m n}(x)-t_{m n}\left(x^{(i)}\right)\right|+\left|t_{m n}\left(x^{(i)}\right)-L^{(i)}\right|+\left|L^{(i)}-L\right|<3 \varepsilon,
$$

for $m \geq m_{0}$ and for all $n$. Hence $x \in V_{\sigma}$. Since $V_{\sigma} \subset V_{\infty}, x \in V_{\infty}$. This completes the proof of the theorem.

Let $A x$ be defined. Then, for all $m, n \geq 0$, we write

$$
t_{m n}(A x)=\sum_{k=1}^{\infty} t(n, k, m) x_{k},
$$

where,

$$
t(n, k, m)=\frac{1}{m+1} \sum_{j=0}^{\infty} a\left(\sigma^{j}(n), k\right),
$$

and $a(n, k)$ denotes the element $a_{n k}$ of the matrix $A$.

\section{Theorem 2}

$A \in\left(l_{\infty}, V_{\infty}\right)$ if and only if

$$
\sup _{m, n} \sum_{k}|t(n, k, m)|<\infty .
$$

\section{Proof}

Sufficiency. Let (7) hold and $x \in l_{\infty}$. Then we have

$$
\begin{aligned}
\left|t_{m n}(A x)\right| & \leq \sum_{k}\left|t(n, k, m) x_{k}\right| \\
& \leq\left(\sum_{k}|t(n, k, m)|\right)\left(\sup _{k}\left|x_{k}\right|\right) .
\end{aligned}
$$

Now, taking the supremum over $m, n$ on both sides, we get $A x \in V_{\infty}$ for $x \in l_{\infty}$, i.e., $A \in\left(l_{\infty}, V_{\infty}\right)$.

Necessity. Let $A \in\left(l_{\infty}, V_{\infty}\right)$. Write $q_{n}(x)=\sup _{m}\left|t_{m n}(A x)\right|$. It is easy to see that for $n \geq 0, q_{n}$ is a continuous seminorm on $l_{\infty}$ and $\left(q_{n}\right)$ is pointwise bounded on $l_{\infty}$. Suppose (7) is not true. Then there exists $x \in l_{\infty}$ with $\sup _{n} q_{n}(x)=\infty$. By the principle of condensation of singularities ${ }^{[5]}$, the set

$$
\left\{x \in l_{\infty}: \sup _{n} q_{n}(x)=\infty\right\}
$$

is of second category in $l_{\infty}$ and hence nonempty, that is, there is $x \in l_{\infty}$ with $\sup _{n}$ $q_{n}(x)=\infty$. But this contradicts the fact that $\left(q_{n}\right)$ is pointwise bounded on $l_{\infty}$. Now, by the Banach-Steinhauss theorem, there is a constant $M$ such that

$$
q_{n}(x) \leq M\|x\|_{1} .
$$


Now define a sequence $x=\left(x_{k}\right)$ by

$$
x_{k}= \begin{cases}\operatorname{sgn} t(n, k, m) & \text { for each } n, m \text { and } 1 \leq k \leq k_{0}, \\ 0 & \text { for } k>k_{0} .\end{cases}
$$

Then $x \in l_{\infty}$. Applying this sequence to (8), we get (7).

This completes the proof of the theorem.

If $\sigma$ is a translation, then by the above theorem, we obtain

\section{Corollary 3}

$A \in\left(l_{\infty}, f_{\infty}\right)$ if and only if

$$
\sup _{m, n} \sum_{k} \frac{1}{m+1}\left|\sum_{j=0}^{m} a_{n+j, k}\right|<\infty \text {. }
$$

\section{Theorem 4}

$A \in\left(l_{1}, V_{\infty}\right)$ if and only if

$$
\sup _{n, k, m}|t(n, k, m)|<\infty .
$$

\section{Proof}

Sufficiency. Suppose that $x=\left(x_{k}\right) \in l_{1}$. We have

$$
\begin{aligned}
\left|t_{m n}(A x)\right| & \leq \sum_{k}\left|t(n, k, m) x_{k}\right| \\
& \leq\left(\sup _{k}|t(n, k, m)|\right)\left(\sum_{k}\left|x_{k}\right|\right) .
\end{aligned}
$$

Taking the supremum over $n, m$ on both sides and using (9), we get $A x \in V_{\infty}$ for $x \in l_{1}$.

Necessity. Let us define a continuous linear functional $Q_{m n}$ on $l_{1}$ by

$$
Q_{m n}(x)=\sum_{k} t(n, k, m) x_{k} \text {. }
$$

Now,

$$
\left|Q_{m n}(x)\right| \leq \sup _{k}|t(n, k, m)|\|x\|_{1}
$$

and hence

$$
\left\|Q_{m, n}\right\| \leq \sup _{k}|t(n, k, m)| .
$$

For any fixed $k \in \mathbb{N}$, define $x=\left(x_{i}\right)$ by

$$
x_{i}= \begin{cases}\operatorname{sgn} t(n, k, m) & \text { for } i=k, \\ 0 & \text { for } i \neq k .\end{cases}
$$


Then $\|x\|_{1}=1$, and

$$
\begin{aligned}
\left|Q_{m n}(x)\right| & =\left|t(n, k, m) x_{k}\right| \\
& =|t(n, k, m)|\|x\|_{1},
\end{aligned}
$$

hence

$$
\left\|Q_{m n}(x)\right\| \geq \sup _{k}|t(n, k, m)| .
$$

By (10) and (11), we get

$$
\left\|Q_{m n}(x)\right\|=\sup _{k} \mid t(n, k, m) .
$$

Since $A \in\left(l_{1}, V_{\infty}\right)$, we have, for $x \in l_{1}$,

$$
\sup _{m, n}\left|Q_{m, n}(x)\right|=\sup _{m, n}\left|\sum_{k} t(n, k, m) x_{k}\right|<\infty .
$$

Hence, by the uniform boundedness principle, we have

$$
\sup _{m, n}\left\|Q_{m, n}(x)\right\|=\sup _{m, n, k}|t(n, k, m)|<\infty .
$$

This complete the proof of the theorem.

If we take $\sigma(n)=n+1$ in the above theorem, we get

\section{Corollary 5}

$A \in\left(l_{1}, f_{\infty}\right)$ if and only if

$$
\sup _{n, k, m} \frac{1}{m+1}\left|\sum_{j=0}^{m} a_{n+j, k}\right|<\infty .
$$

\section{References}

[1] Lorentz, G.G., A contribution to the theory of divergent sequences, Acta Math., 80: 167190 (1948).

[2] Mursaleen, On some new invariant matrix method of summability, Quart. J. Math. Oxford, 34: (2) 77-86 (1983).

[3] Yosida, K., Functional Analysis, Springer Verlag, Berlin, Heidelberg, New York (1971).

[4] Mursaleen, Gaur, A.K. and Chishti, T.A., On some new sequence spaces of invariant means, Acta Math. Hung., 75: 209-214 (1997).

[5] Schaefer, P., Infinite matrices and invariant means, Proc. Amer. Math. Soc., 36: 104-110 (1972). 


$$
\text { محول المصفوفات إلى فراغ متسلسلات جديد }
$$

$$
\begin{aligned}
& \text { عدنان الحميدان } \\
& \text { قسم الرياضيات ، كلية العلوم ، جامعة الملك عبدالعزيز }
\end{aligned}
$$

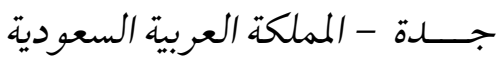

المستخلص. في هذا البحث تم تعريف الفراغ مب من خلال مفهوم

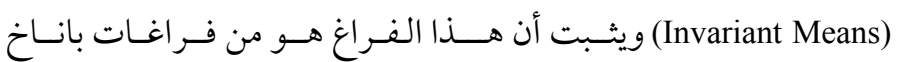
(Banach Space) 